\title{
Pharmacokinetics, pharmacodynamics and metabolism of a novel anticancer agent for prostate cancer
}

\author{
JUN YANG ${ }^{1}$, SUNJOO AHN ${ }^{1,3}$, ZENGRU WU $^{1}$, DONG-JIN HWANG ${ }^{2}$, \\ DUANE D. MILLER ${ }^{2,3}$ and JAMES T. DALTON ${ }^{1,3}$ \\ ${ }^{1}$ Division of Pharmaceutics, College of Pharmacy, The Ohio State University, Columbus, OH 43210; \\ ${ }^{2}$ Department of Pharmaceutical Sciences, College of Pharmacy, The University of Tennessee Health Science Center, \\ Memphis, TN 38163; ${ }^{3}$ Preclinical Research and Development, GTx, Inc., Memphis, TN 38163, USA
}

Received February 17, 2012; Accepted March 29, 2012

DOI: 10.3892/ijo.2012.1442

\begin{abstract}
The objective of these studies was to examine the murine pharmacokinetics, pharmacodynamics and metabolism of (3-(1H-indol-2-yl)phenyl)(1H-indol-2-yl)methanone (Indole 15), a novel tubulin inhibitor for the treatment of cancer. We developed HPLC and LC/MS/MS assays to quantitate Indole 15 and characterize its metabolites in vivo. Pharmacokinetic studies were performed after intravenous (IV), oral (PO) and subcutaneous (SC) administration of $10 \mathrm{mg} / \mathrm{kg}$ doses to male ICR mice. Urine and fecal samples were also collected over a 72-h period to identify metabolites. Pharmacodynamic studies were conducted by monitoring the tumor size change during a period of two weeks in PC-3 tumor bearing mice after daily IV administration of Indole 15 at doses of 0,10,50,100 and $150 \mathrm{mg} / \mathrm{kg}$. The pooled plasma concentration data after administration via different dose routes was simultaneously fitted by a two-compartmental model. Indole 15 was moderately well absorbed after PO and SC administration, with a bioavailable fraction of 0.27 and 0.72 , respectively. The steady state volume distribution $\left(V_{s s}\right)$ and clearance $(C L)$ were estimated to be $7.01 / \mathrm{kg}$ and $4.36 \mathrm{l} / \mathrm{h} / \mathrm{kg}$, respectively. The mean data of PC-3 tumor growth in mice was fitted well by a transduction model using fixed plasma pharmacokinetics as a driving function. Analysis of the metabolites in mice indicated that the compound undergoes extensive oxidative metabolism with subsequent sulfation. These studies demonstrate that favorable pharmacokinetic and pharmacodynamic properties of Indole 15 offer hope for achieving pharmacological activity in early clinical trials.
\end{abstract}

Correspondence to: Dr James T. Dalton, Preclinical Research and Development, GTx Inc., 3 North Dunlap St., Memphis, TN 38163, USA

E-mail: jdalton@gtxinc.com

Key words: pharmacokinetics, pharmacodynamics, metabolism, anticancer, Indole 15

\section{Introduction}

Microtubule-interacting drugs are important agents in cancer chemotherapy. Such drugs are commonly classified into two major categories: microtubule-stabilizing (e.g., taxanes) and microtubule-destabilizing agents (e.g., vinca alkaloids and colchicine). The potency, efficacy and widespread clinical use of these agents in a variety of cancers stand testament to the importance of tubulin and its role in cancer growth. Unfortunately, these drugs also share a common mechanism of drug resistance, namely P-glycoprotein or ATP binding cassette (ABC) transporter protein-mediated drug efflux, which limits their efficacy in many tumors. Therefore, new anticancer drugs that can overcome or circumvent the multidrug resistance represent an important field in drug discovery.

We recently reported promising in vitro and in vivo anticancer activity of a novel bis-indole agent [Indole 15; (3-(1H-indol-2-yl)phenyl)(1H-indol-2-yl)methanone] (1). This novel agent inhibits tubulin polymerization and arrests the cell cycle in $\mathrm{G}_{2}-\mathrm{M}$ phase (1). It exhibits broad antitumor activity in a variety of cancer cells with $\mathrm{IC}_{50}$ values ranging from 34-162 nM and retained its potent anticancer activity in cancer cell lines that overexpress P-glycoprotein, multidrug resistance associated proteins (MRP) and breast cancer-resistance protein (BCRP). Indole 15 also demonstrated the ability to inhibit PC-3 tumor xenograft growth as well as docetaxel and vinblastine, identifying it as a novel chemotherapeutic agent that can overcome taxane-resistant cancer induced by multidrug resistance.

Herein, we report the in vivo pharmacokinetics, pharmacodynamics and metabolism of Indole 15 in mice. Characterization of the pharmacokinetics, pharmacodynamics and metabolic profile of Indole 15 provided us with a better understanding of the factors affecting the in vivo efficacy of this chemical scaffold to direct future structure modification $(1,2)$.

\section{Materials and methods}

Chemicals and reagents. (3-(1H-indol-2-yl)phenyl)( $1 H$-indol2-yl)methanone (Indole 15) and its internal standard (Fig. 1) were synthesized and characterized in our laboratories as previously described (1). The purities of synthesized compounds were confirmed by NMR, elemental analysis and mass spectrometry. 


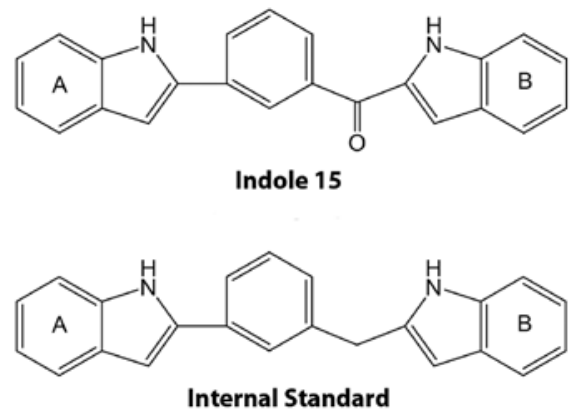

Figure 1. Chemical structure of Indole 15 and its internal standard. Indole 15 or (3-(1H-indol-2-yl)phenyl)( $1 H$-indol-2-yl)methanone and internal standard or 2-(3-(1H-indol-2-yl)benzyl]-1 $H$-indole.

HPLC grade acetonitrile and ethyl acetate were purchased from Fisher Scientific Company (Fair Lawn, NJ, USA). Pooled liver microsomes from mice, rats, dogs, monkeys, and humans were purchased from Xenotech (Lenexa, KS, USA). Nicotinamide adenine dinucleotide phosphate (NADPH) regenerating system was purchased from BD (Franklin Lakes, NJ, USA). All other chemicals were purchased from Sigma Chemical Company (St. Louis, MO, USA).

Animals. Male ICR mice were purchased from Harlan Biosciences (Indianapolis, IN, USA). Male Balb/c mice weighing 19-22 g were purchased from the National Cancer Institute (Bethesda, MD, USA). All animals were maintained in a special facility with a 12-h light/dark cycle with food and water available ad libitum. All animal studies were approved by the Institutional Animal Care and Use Committee at The Ohio State University.

Pharmacokinetic studies. Male ICR mice weighing about $20 \mathrm{~g}$ were used for pharmacokinetic studies. A single dose of $10 \mathrm{mg} /$ $\mathrm{kg}$ dose was administered into mice via the intravenous (IV), subcutaneous (SC) and oral (PO) route. Blood samples were collected prior to the dose and at 5, 10, 20, 30, 40, 60, 90, 120, $240,480,720,1,080$ and 1,440 min after drug administration. At each time point, three to four mice were euthanized by $\mathrm{CO}_{2}$ inhalation and blood samples (up to $600 \mu \mathrm{l}$ each) were taken from the posterior vena cava. Blood samples were immediately centrifuged at $3,000 \mathrm{xg}$ for $10 \mathrm{~min}$. Two hundred microliters of plasma from each sample was transferred to a clean plastic tube and stored at $-80^{\circ} \mathrm{C}$ until analysis.

Sample preparation for HPLC-UV analysis in mouse pharmacokinetics. A $100 \mu \mathrm{l}$ aliquot of each plasma sample was spiked with $10 \mu \mathrm{l}$ of an internal standard (a structural analog of Indole $15,7.5 \mu \mathrm{g} / \mathrm{ml}$ final concentration) and mixed well with $1 \mathrm{ml}$ of acetonitrile. After centrifugation at $12,000 \times \mathrm{g}, 4^{\circ} \mathrm{C}$ for $5 \mathrm{~min}$, the supernatant was collected and evaporated. The residues were reconstituted in $150 \mu \mathrm{l}$ of mobile phase and an aliquot of $100 \mu \mathrm{l}$ was used for HPLC analysis.

HPLC-UV analysis. The separation of Indole 15 and internal standard was carried out using an isocratic mobile phase (acetonitrile $/ \mathrm{H}_{2} \mathrm{O}: 55 / 45, \mathrm{v} / \mathrm{v}$ ) at a flow rate of $1 \mathrm{ml} / \mathrm{min}$ with a Nova-Pak $C_{18}$ column (3.9x150 mm, particle size $4 \mu \mathrm{m}$ ) (Waters Corporation, Milford, MA, USA). The UV absorbance of eluents was monitored at $244 \mathrm{~nm}$. Calibration standards were prepared in blank mouse plasma with a linear range of 0.02 to $20 \mu \mathrm{g} / \mathrm{ml}$ and intra- and inter-day coefficients of variation at all concentrations were less than $6 \%$. The recovery of compound ranged from $86.9-90.1 \%$, as measured using low, medium and high concentrations of samples. No endogenous interfering peak was found in the blank plasma at the retention time of either Indole 15 or the internal standard.

In vitro metabolism studies. For both phase I and phase I \& II in vitro metabolism studies, the incubation mixture consisted of $1 \mathrm{mg} / \mathrm{ml}$ liver microsomal proteins, $3 \mathrm{mM}$ NADPH, and $0.5 \mu \mathrm{M}$ test compound in $65 \mathrm{mM}$ potassium phosphate buffer ( $\mathrm{pH}$ 7.4). For phase I \& II in vitro metabolism studies, $5 \mathrm{mM}$ uridine diphosphate glucuronic acid (UDPGA), $5 \mathrm{mM} D$-saccharolactone, $50 \mu \mathrm{g} / \mathrm{ml}$ alamethicin, and $3 \mathrm{mM}$ magnesium chloride were added to the phase I metabolism study mixture. The final concentration of methanol (used for dissolving the substrate) was $1 \%(\mathrm{v} / \mathrm{v})$. Total volume of the incubation was $200 \mu \mathrm{l}$ and the reaction mixtures were incubated at $37^{\circ} \mathrm{C}$. To generate the metabolic stability curves for Indole 15 , separate incubations were stopped at 10, 20,30,60, and 90 min for analysis of the concentration of Indole $15(\mathrm{n}=3)$. All reactions were stopped by the addition of $200 \mu \mathrm{l}$ ice-cold acetonitrile containing the internal standard to a $100 \mu \mathrm{l}$ sample of the reaction mixture. Subsequently, the samples were then centrifuged at $3,000 \mathrm{x} g$ for 5 min and supernatant was analyzed.

Pharmacodynamic studies. Cultured PC-3 prostate cancer cells were detached by trypsinization and resuspended in $0.9 \%$ saline. Two million cells suspended in $200 \mu \mathrm{l}$ of saline were injected subcutaneously into both flanks of Balb/c mouse $(n=15)$. Daily treatment (5 days on/2 days off) was initiated with Indole 15 (10, 50,100 or $150 \mathrm{mg} / \mathrm{kg} / \mathrm{day}$ ) via tail vein when tumors reached a volume of approximately $175 \mathrm{~mm}^{3}$. Body weight and tumor size were measured every other day and tumor volume was calculated as $\mathrm{V}=\pi / 6 \cdot(\text { length).(width) })^{2}(1,3)$. Tumor mass was calculated as $\mathrm{R}=\mathrm{V}^{*} \mathrm{Q}$, assuming density $\mathrm{Q}=1 \mathrm{mg} / \mathrm{mm}^{3}$ for tumor tissue (4).

Pharmacokinetic and pharmacodynamic data analysis. The pharmacokinetic data analysis was based on a naive pooled approach. Data were simultaneously fitted by a two-compartment linear model:

$$
\begin{gathered}
\frac{d A_{1}}{d t}=-K_{a} \cdot A_{1} \\
V_{c} \cdot \frac{d C_{1}}{d t}=K_{a} \cdot A_{1}-C L \cdot C_{1}+C L_{d} \cdot\left(C_{2}-C_{1}\right) \\
V_{2} \cdot \frac{d C_{2}}{d t}=C L_{d} \cdot\left(C_{1}-C_{2}\right)
\end{gathered}
$$

where $A_{l}$ and $k_{a}$ are the depot compartment and absorption rate, respectively. $V_{c}$ and $C_{l}$ are central compartment volume and concentration, $V_{2}$ and $C_{2}$ are peripheral compartment volume and concentration, and $C L$ and $C L_{d}$ represent central compartment and intercompartment clearance, respectively.

Mean values of tumor size were modeled by a transduction model described previously $(4,5)$ with the assumption that the effect of drug is to shunt cycling cells into a successive series of compartments after which the cells are dead (6). A schematic 
representation of the model is shown in Fig. 2. Tumor mass thus consists of the residual cycling cells plus cells that are committed to death but not yet dead. Differential equations and initial conditions of the transduction model are as follows:

$$
\begin{array}{ccc}
\frac{d R_{1}}{d t}=g\left[R_{1}, w(t)\right]-k \cdot C_{p} \cdot R_{1}, & R_{1}(0)=w(0) & (\mathrm{Eq} 4) \\
\frac{d R_{2}}{d t}=k \cdot C_{p} \cdot R_{1}-R_{2} / \tau, & R_{2}(0)=0 \\
\frac{d R_{3}}{d t}=\left(R_{2}-R_{3}\right) / \tau, & R_{3}(0)=0
\end{array}
$$

where $R_{l}$ represents cycling cells, $g\left[R_{l}, w(t)\right]$ is the growth function of cycling cells, $C_{p}$ is plasma concentration of drug, $\tau$ is mean transit time, $k$ is the second-order killing constant, $R_{l}(0)$ is the initial tumor size or number of proliferating cells, and $R_{2}, R_{3}$ refers to the cell transduction compartments; their initial conditions of $R_{2}, R_{3}$ are all zero. Tumor size, $w(t)$, is the sum of all the compartments; $w(t)=\sum R_{i}$. The tumor growth, $g\left[R_{l}, w(t)\right]$, was modeled by a first order growth function, $g\left[R_{l}, w(t)\right]=K_{g} \cdot R_{l}$, which was described by Yang et al (6).

The variance models for both pharmacokinetics and pharmacodynamics were defined as the followings:

$$
\sigma^{2}=\left(\sigma_{1}+\sigma_{2} \cdot \hat{Y}\right)^{2}
$$

where $\sigma_{1}$ and $\sigma_{2}$ are the variance parameters, and $\hat{Y}$ represents model predicted value of drug concentration or tumor size.

The goodness of fit was assessed by model convergence, visual inspection, precision of the parameter estimates, Akaike Information Criteria (AIC), Schwarz Criteria (SC), and examination of residuals. The maximum likelihood (ML) method was applied for both pharmacokinetics and pharmacodynamics parameter estimation using ADAPT II (7).

In vivo metabolism studies. Before dosing, urine and feces were collected for $24 \mathrm{~h}$ as blank samples. The mice were then administered Indole 15 via the tail vein at a dose of $10 \mathrm{mg} / \mathrm{kg}$. Urinary and fecal samples were collected at 24-h intervals for up to $72 \mathrm{~h}$ after IV administration. The animals were transferred to new cages at the end of each collection interval. All samples were stored at $-80^{\circ} \mathrm{C}$ until analysis.

Before analysis, urine samples were thawed and extracted with two volumes of ethyl acetate. The extraction procedure was repeated three times and the combined organic phase and the aqueous phase were evaporated separately to dryness. The residues were reconstituted in a mobile phase of acetonitrile $/ \mathrm{H}_{2} \mathrm{O}$ [55:45 (v/v)], and the solution was filtered through an Acrodisc syringe filter $(0.2 \mu \mathrm{m}, 13 \mathrm{~mm}$; Pall Corporation, East Hills, NY, USA). The filtrate was applied to liquid chromatography tandem mass spectrometry (LC-MS/MS) analyses as described below. Fecal samples were extracted three times with $10 \mathrm{ml}$ of a mixture of methanol/ethyl acetate [2:1 (v/v)]. The liquid phase after each extraction was combined and evaporated. The resulting semisolid residues were then mixed with methanol and were filtered followed by LC-MS/MS analyses.

LC-MS/MS analysis. Mass spectrometric analyses were carried out using a ThermoFinnigan LCQ DECA XP MAX

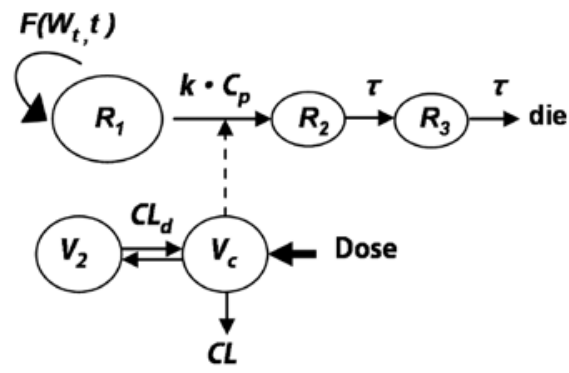

Figure 2. A cell distribution model using transduction process to describe the chemotherapeutic system of Indole 15.

ion trap mass spectrometer (San Jose, CA, USA) coupled with a Surveyor (ThermoQuest, San Jose, CA, USA) HPLC system consisting of an autosampler (SIL-20A) and an MS quaternary pump with on-line degasser. The autosampler was set at $4^{\circ} \mathrm{C}$ during analysis. Data acquisition was controlled by Xcalibur software. An aliquot (10 $\mu \mathrm{l})$ of each sample was injected into a Beckman Coulter ODS column $(250 \times 2.0 \mathrm{~mm}, 5 \mu \mathrm{m})$ at a flow rate of $0.2 \mathrm{ml} / \mathrm{min}$ using a gradient mobile phase comprised of water (A) and acetonitrile (B). The mobile phase was comprised of a $20 \%$ of B for the first 5 min and the flow was diverted to waste, increased to $65 \%$ of $\mathrm{B}$ in a linear gradient from 5 to $35 \mathrm{~min}$, and then further increased to $90 \%$ of B from 35 to $50 \mathrm{~min}$ and maintained for $5 \mathrm{~min}$, and finally returned to $20 \% \mathrm{~B}$ at $70 \mathrm{~min}$. The column was equilibrated with the initial mobile phase for $10 \mathrm{~min}$. The mass spectrometer was tuned with Indole 15 and operated in either positive or negative mode. The heated capillary temperature was set at $230^{\circ} \mathrm{C}$, and spray voltages were set at 3.0 and $3.5 \mathrm{kV}$ for positive and negative ion mode, respectively. The sheath and auxiliary gas flow rates were 60 and $20 \mathrm{ml} / \mathrm{min}$, respectively. For MS/MS analysis, selected precursor ions were isolated with a width of $3 \mathrm{~m} / \mathrm{z}$ and fragmented with $35 \%$ energy for positive ion mode and $38 \%$ for negative ion mode.

Statistical analysis. Statistical analyses were performed using single-factor analysis of variance (ANOVA). p $<0.05$ was considered as a statistically significant difference.

\section{Results}

Pharmacokinetics of Indole 15 in mice. The pharmacokinetics of Indole 15 were examined in ICR mice. A single dose $(10 \mathrm{mg} / \mathrm{kg})$ of Indole 15 via various routes of administration (IV, PO and SC) was studied in order to approximate its in vivo disposition and interpret the results of forthcoming in vivo xenograft studies.

Overall the plasma concentration profiles were fitted very well by a simultaneous two-compartment model (Fig. 3). Plasma concentrations of Indole 15 declined rapidly after IV injection, with a terminal half-life of $2.97 \mathrm{~h}$. Central volume $(V c)$ and clearance $(C L)$ were estimated to be $3.67 \mathrm{l} / \mathrm{kg}$ and $4.36 \mathrm{l} / \mathrm{h} /$ $\mathrm{kg}$, respectively (Table I). Plasma concentrations of Indole 15 peaked at about 2-3 h after SC or PO administration, with absolute bioavailability of 71.9 and $27.4 \%$, respectively. The terminal phase after PO and SC administration prolonged as compared to that observed after an IV dose, likely reflecting a 


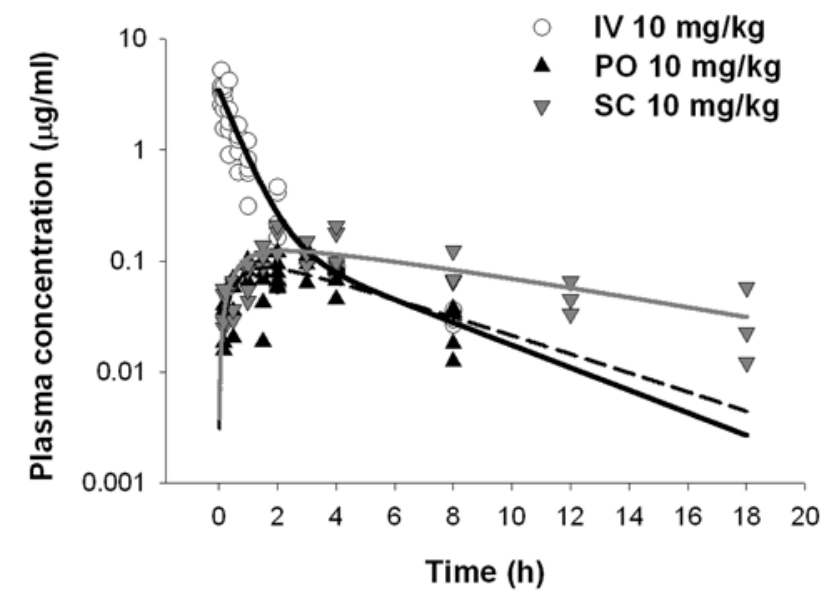

Figure 3. Pooled plasma concentration-time profile of Indole 15 in mice. $10 \mathrm{mg} / \mathrm{kg}$ of Indole 15 was administered through IV, SC and PO routes to individual mice. Blood sampling was drawn at different time points $(\mathrm{n}=5)$ until $24 \mathrm{~h}$ post-dosing.

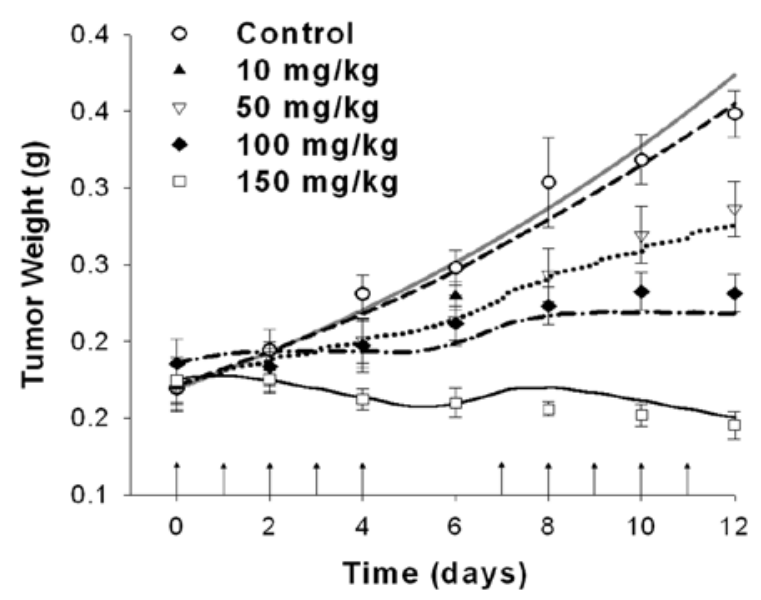

Figure 4. Model predictions of IV bolus of Indole 15 on the time course of PC-3 tumor growth in mice. Mean \pm SD, $n=4-6$.

slow absorption from the PO and SC injection sites. No parent drug was detectable in either urinary or fecal samples after IV administration of Indole 15. These data, coupled with estimates of the hepatic blood flow of the mouse $(5.4 \mathrm{l} / \mathrm{h} / \mathrm{kg})(8)$, suggest that Indole 15 was extensively metabolized in the liver with a high hepatic extraction.

Pharmacodynamic model for tumor growth in mice. The antitumor activity of Indole 15 was evaluated in mice bearing a PC-3 xenograft over a period of 2 weeks. Compared to the vehicle control group, the tumor size at end of treatment decreased in a dose-dependent manner, with a complete inhibition of tumor growth at the highest dose $(150 \mathrm{mg} / \mathrm{kg}$ ) (Fig. 4). The body weight changes among different dose cohorts at the end of treatment were not significantly different (data not shown), suggesting that Indole 15 was well tolerated in tumor-bearing mice.

Assuming that the pharmacokinetics of Indole 15 in mice did not change with dose, the PC-3 tumor growth in mice was well described by a pharmacodynamic transduction model using fixed pharmacokinetics as a driving force for drug exposure.
Table I. Pharmacokinetic parameters of Indole $15(10 \mathrm{mg} / \mathrm{kg})$ in ICR mice.

\begin{tabular}{lcc}
\hline Parameters & Estimate & CV $(\%)$ \\
\hline$k_{\alpha_{-} p o}(1 / \mathrm{h})$ & 0.231 & 14.4 \\
$k_{\alpha_{-} s c}(1 / \mathrm{h})$ & 0.109 & 11.4 \\
$V_{c}(1 / \mathrm{kg})$ & 3.67 & 9.6 \\
$V_{2}(1 / \mathrm{kg})$ & 3.33 & 25.0 \\
$C L_{d}(1 / \mathrm{h} / \mathrm{kg})$ & 1.01 & 21.4 \\
$C L(1 / \mathrm{h} / \mathrm{kg})$ & 4.36 & 5.8 \\
$F_{p o}$ & 0.274 & 11.2 \\
$F_{s c}$ & 0.719 & 9.7 \\
$\sigma_{I}$ & 0 & Fixed \\
$\sigma_{2}$ & 0.381 & 7.5 \\
\hline
\end{tabular}

Parameters were estimated by simultaneous two-compartmental analysis.

Table II. Pharmacodynamic parameters from tumor growth transduction model.

\begin{tabular}{lcc}
\hline Parameters & Estimate & CV\% \\
\hline$\tau(\mathrm{d})$ & 0.532 & 38.4 \\
$k_{g}(1 / \mathrm{d})$ & 0.066 & 2.7 \\
$k[1 /(\mathrm{mg} \cdot \mathrm{d})]$ & 0.070 & 6.2 \\
$\sigma_{1}$ & 0 & Fixed \\
$\sigma_{2}$ & 0.043 & 12.5 \\
\hline
\end{tabular}

Table III. Half-life of Indole 15 in mouse, rat, dog, monkey, and human liver microsomes.

\begin{tabular}{lcc}
\hline & \multicolumn{2}{c}{ Half-life (min) } \\
\cline { 2 - 3 } & Phase I & Phase I \& II \\
\hline Mouse & 41 & 34 \\
Rat & $>90$ & $>90$ \\
Dog & 32 & 35 \\
Monkey & 22 & 25 \\
Human & 43 & 59 \\
\hline
\end{tabular}

The initial tumor weight in each dose group was fixed as the measured mean value. Pharmacodynamic parameter estimates are presented in Table II, and demonstrate a reasonable approximation of the observed tumor growth data (Fig. 4).

Metabolic stability of Indole 15 in mouse, rat, dog, monkey, and human liver microsomes. Metabolic stability was investigated by incubating Indole 15 with liver microsomes from mouse, rat, dog, monkey, and human. In order to evaluate the metabolism by phase II conjugation, UDPGA was introduced to the microsomal preparation. Half-life was determined with a two parameter single exponential decay curves using SigmaPlot Version 10.0 (Systat Software Inc., Chicago, IL, USA). Metabolic stability 
Table IV. Indole 15 and its major urinary and fecal metabolites found in mice.

\begin{tabular}{|c|c|c|c|}
\hline Molecular Ions & Polarity & $\begin{array}{l}\text { MS }^{2} \text { Product ions } \\
\qquad(\mathrm{m} / z)\end{array}$ & $\begin{array}{c}\text { Assigned structure and } \\
\text { proposed fragmentation patterns }\end{array}$ \\
\hline 337 (Indole 15) & {$[\mathrm{M}+\mathrm{H}]^{+}$} & $220,192\left(\mathrm{MS}^{3}\right)$ & \\
\hline 353 (Indole $15+\mathrm{O}$ ) & {$[\mathrm{M}+\mathrm{H}]^{+}$} & 236,220 & \\
\hline 369 (Indole $15+2 \mathrm{O})$ & {$[\mathrm{M}+\mathrm{H}]^{+}$} & 236 & 36 \\
\hline 431 (Indole $15+\mathrm{O}+\mathrm{SO}_{3} \mathrm{H}$ ) & {$[\mathrm{M}-\mathrm{H}]^{-}$} & 351 & \\
\hline 447 (Indole $\left.15+2 \mathrm{O}+\mathrm{SO}_{3} \mathrm{H}\right)$ & {$[\mathrm{M}-\mathrm{H}]^{-}$} & 367 & \\
\hline 527 (Indole $\left.15+2 \mathrm{O}+2 \mathrm{SO}_{3} \mathrm{H}\right)$ & {$[\mathrm{M}-\mathrm{H}]^{-}$} & 447 & \\
\hline 463 (Indole $15+3 \mathrm{O}+\mathrm{SO}_{3} \mathrm{H}$ ) & {$[\mathrm{M}-\mathrm{H}]^{-}$} & 383 & \\
\hline 543 (Indole $15+3 \mathrm{O}+2 \mathrm{SO}_{3} \mathrm{H}$ ) & {$[\mathrm{M}-\mathrm{H}]^{-}$} & 463 & 463 \\
\hline
\end{tabular}

of Indole 15 in the presence and absence of UDPGA was not significantly different as evidenced by similar half-lives during incubation with phase I and phase I \& II systems (Table III), suggesting that glucuronidation of Indole 15 is not a predominant metabolite pathway. Indole 15 was most stable in rat liver microsomes with the longest half-life ( $>90 \mathrm{~min}$ ) and degraded quickly in monkey liver microsomes with the shortest half-life (22 $\mathrm{min}$ ) in phase I metabolism (Table III). The phase I metabolic stability of Indole 15 in human liver microsomes (43 min) appeared to be more similar to that of mouse than the other three species (Table III).

Identification of metabolites of Indole 15 in urine and feces. Urinary and fecal samples were collected at 24-h intervals for up to $72 \mathrm{~h}$ after IV administration of Indole 15 in mice. The identification of major metabolites was based on the MS/MS data and the chromatographic retention time. Parent drug was not identified in either urine or feces, indicating that Indole 15 was extensively metabolized in mice. Fig. 5 shows the positive ion mass spectrum of the parent compound (molecular weight of 336). The corresponding $[\mathrm{M}+\mathrm{H}]^{+}$ion $(\mathrm{m} / \mathrm{z} 337)$ yielded a prominent fragment ion of 220 (Fig. 5A), with a neutral loss of $117 \mathrm{Da}$ (the mass of an indole ring). The $\mathrm{MS}^{3}$ of the ion 220 (Fig. 5B) yielded a prominent fragment of 192 with a loss of $28 \mathrm{Da}$ (Fig. 5B), suggesting the fragment ion of 220 is most likely from the breakage of the link between indole ring $\mathrm{B}$ and ketone.
Major metabolites of Indole 15 corresponding to $\mathrm{m} / \mathrm{z} 353$, 369, 431, 447, 463, 527, and 543 were identified (Table IV). The sulfate conjugation products had better MS response in negative ion mode than in positive ion mode. The $\left[\mathrm{M}-\mathrm{H}^{-}\right.$ at $\mathrm{m} / \mathrm{z} 431$ (sulfate conjugate) yielded an ion of $\mathrm{m} / \mathrm{z} 353$ (Indole $15+\mathrm{O})$ with a loss of $80 \mathrm{Da}\left(-\mathrm{SO}_{3}\right.$ moiety). Using a scan filter of MS/MS at 353, we found three chromatographic peaks with the retention time of $34.6,36.5$ and $40.0 \mathrm{~min}$ in fecal samples (Fig. 6A). Further MS/MS fragmental ions at $\mathrm{m} / \mathrm{z}, 236$ and 220 (Fig. 6B and C) suggested that the oxidation of Indole 15 occured at multiple positions. The oxidation position was not determined in the current MS analysis. The product ion at $m / z 369\left([\mathrm{M}+\mathrm{H}]^{+}\right)$corresponds to doublyoxidized Indole 15 (Table IV).

The parent ion at $m / z 447$ ([M-H] $]^{-}$) corresponded to doublyoxidized Indole 15 with mono-sulfate conjugate. This [M-H] ${ }^{-}$ion yielded a prominent product ion at $\mathrm{m} / \mathrm{z} 367$ with an exact loss of a $\mathrm{SO}_{3}$ moiety (80 Da). Again, four chromatographic peaks were identified using this scan filter at 11.7, 13.5, 15.9 and 20.2 min (data not shown). These results confirmed that the oxidations occur at multiple positions, and each isomer could further form a sulfate conjugate. The parent ion at $m / z, 527\left([\mathrm{M}-\mathrm{H}]^{-}\right)$corresponded to oxidized Indole 15 with two sulfate conjugates (Indole $15+2 \mathrm{O}+2$ $\left.\mathrm{SO}_{3} \mathrm{H}\right)$ (Table IV). Similarly, the ions at $m / z 463[\mathrm{M}-\mathrm{H}]^{-}$and $\mathrm{m} / \mathrm{z} 543[\mathrm{M}-\mathrm{H}]^{-}$corresponded to triple-oxidized Indole 15 with mono- and double-sulfate conjugates, respectively. 
342

YANG et al: PHARMACOKINETICS, PHARMACODYNAMICS AND METABOLISM OF INDOLE 15

A

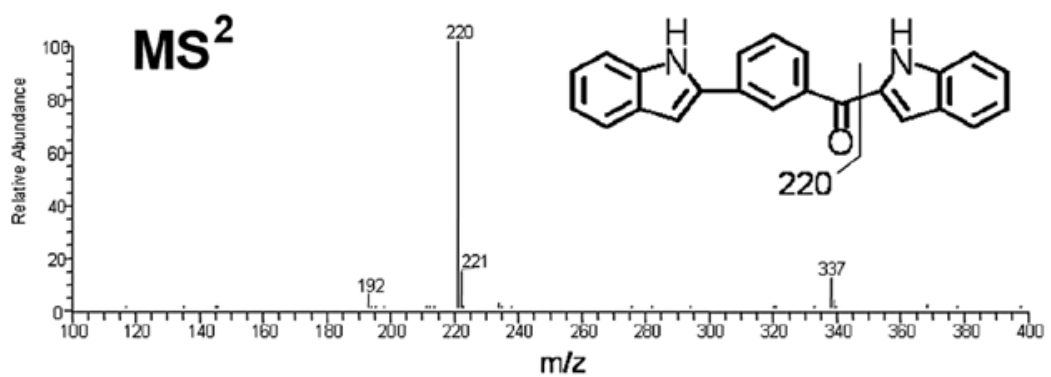

B

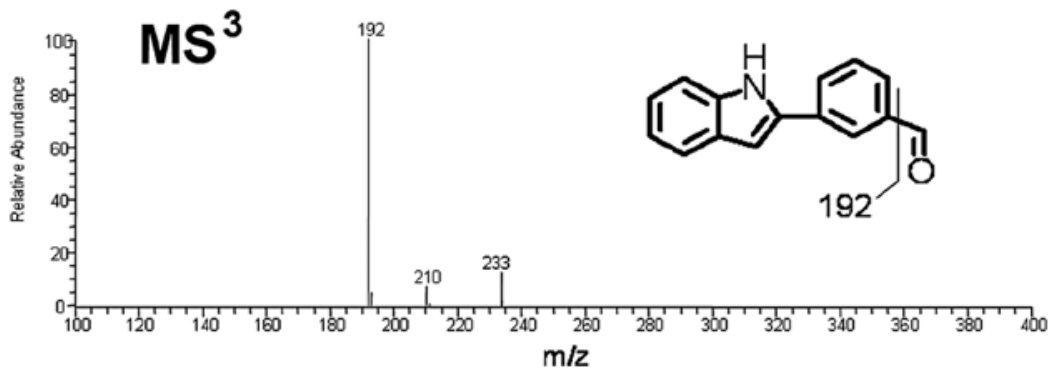

Figure 5. Indole 15 fragmentation mass spectra (m/z 337). (A) MS2. (B) MS3.

A

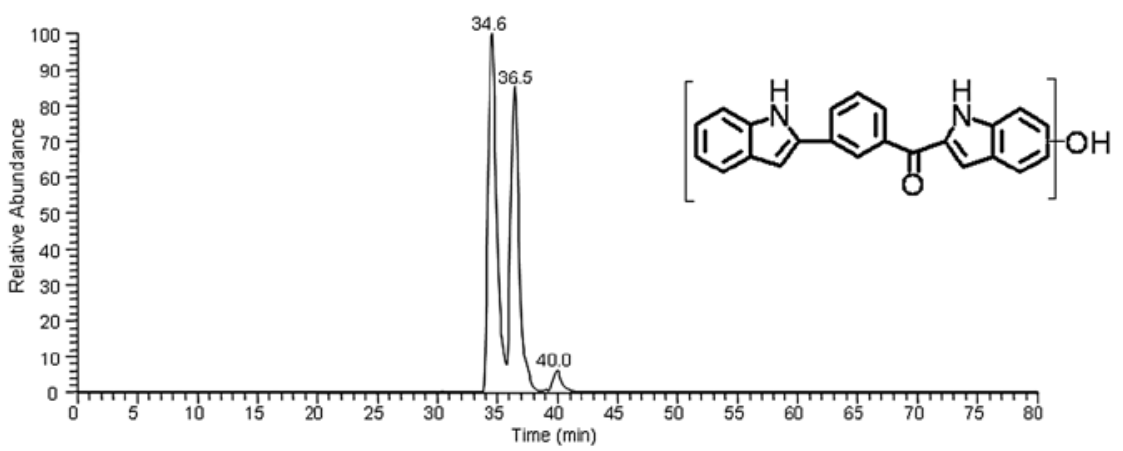

B
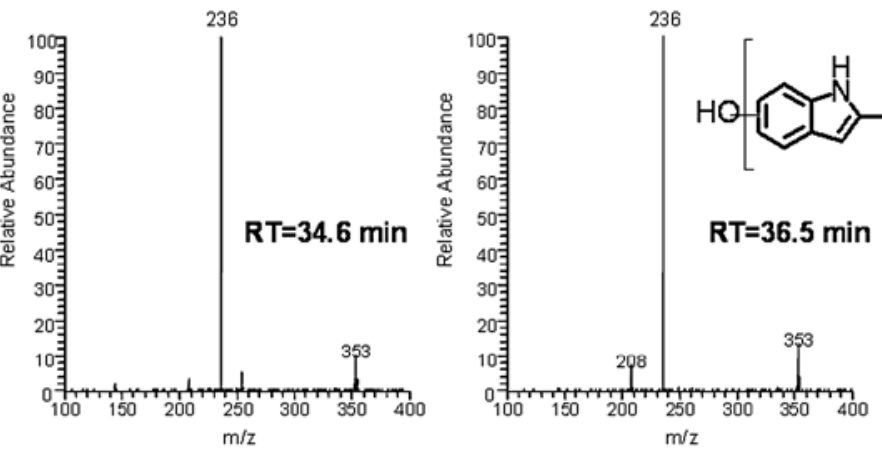

C

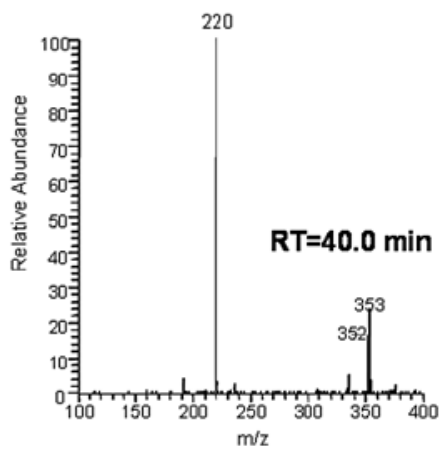

Figure 6. LC/MS/MS chromatogram and spectra of m/z 353. Indicates that the oxidation of Indole 15 occurred at multiple positions. 


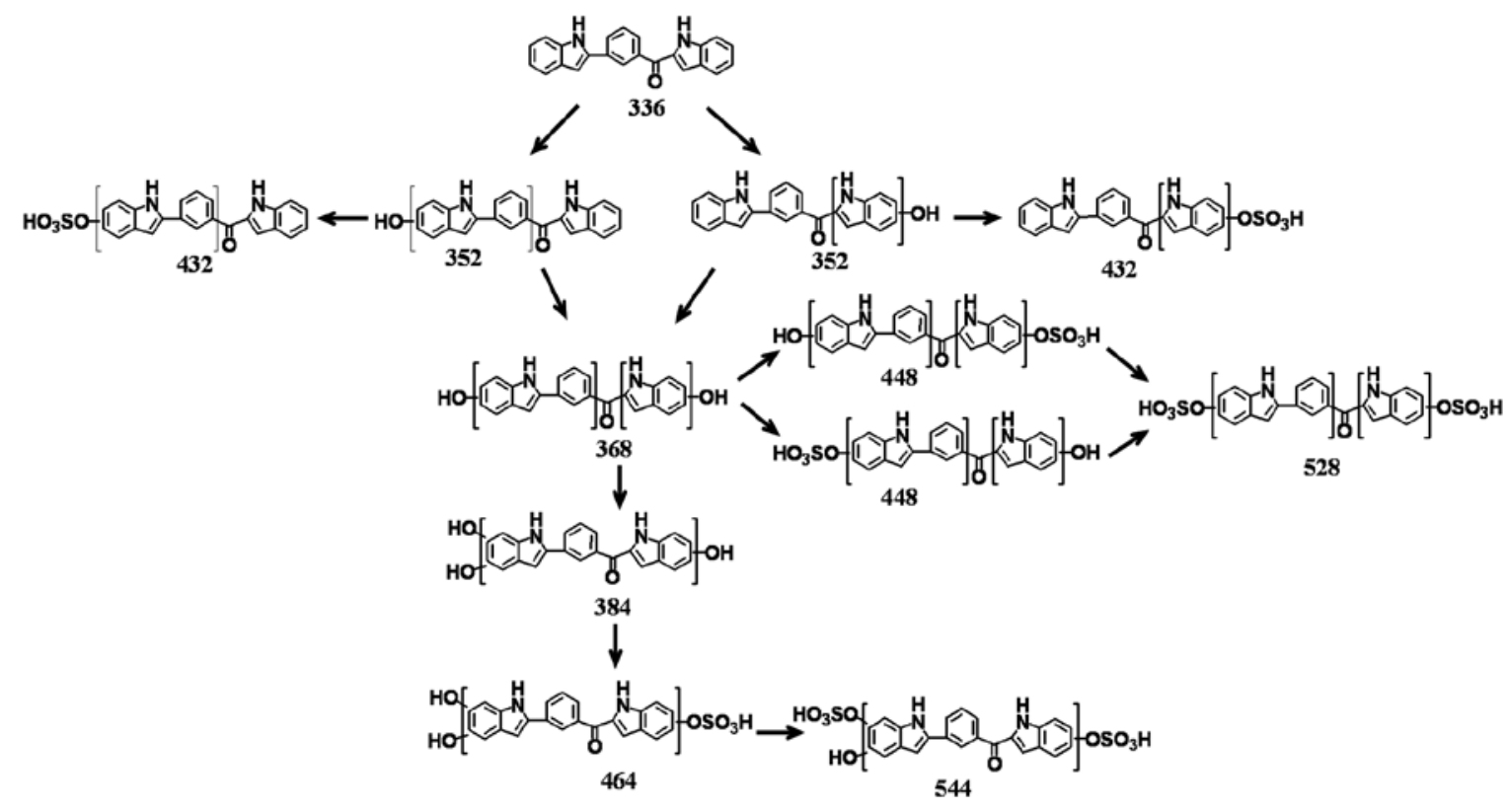

Figure 7. Proposed metabolism of Indole 15 in mice.

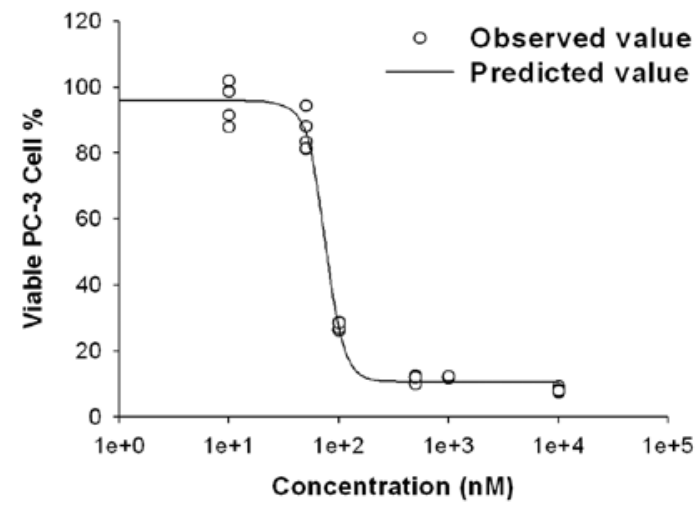

Figure 8. In vitro cell growth inhibition study in PC-3 cells ( $\mathrm{n}=5$ per concentration). PC-3 cell growth was fitted by an inhibitory effect equation, $\left.I=I_{\max }-\left(I_{\max }-I_{0}\right) \cdot\left(C^{\gamma} / I C_{50}{ }^{\gamma}+C^{\gamma}\right)\right)$, where $I_{\max }, I_{0}, I C_{50}$ and $\gamma$ represent the maximum inhibitory effect, drug effect at time zero, concentration that requires to produce half of the maximum inhibitory effect and shape of the curve, respectively. $\mathrm{C}$ represents the concentration of tested drug in the incubation medium.

Other possible metabolism pathways in both positive and negative ion mode were also examined. No glucuronide conjugates or metabolites associated with the ketone reduction were identified. In summary, Indole 15 underwent extensive oxidative hydroxylation with subsequent sulfation. A possible metabolic scheme for the metabolism of Indole 15 in mice is proposed and illustrated in Fig. 7.

\section{Discussion}

Pharmacokinetic, pharmacodynamic, and metabolism studies of Indole 15 have not been previously reported. The studies reported herein demonstrate that Indole 15 is extensively metabolized in mice with a half-life comparable to paclitaxel $(9,10)$. In the in vitro metabolism study using animal liver microsomes, Indole 15 showed similar stability in human and mouse liver
Table V. Parameter estimates for in vitro PC-3 cell growth inhibitory study.

\begin{tabular}{lcccc}
\hline Parameters & Estimate & SE & CV \% & $95 \%$ CI \\
\hline $\mathrm{I}_{\max }$ & 0.959 & 0.016 & 1.67 & $0.926-0.990$ \\
$\mathrm{I}_{0}$ & 0.106 & 0.009 & 8.49 & $0.088-0.124$ \\
$\mathrm{IC}_{50}$ & 75.3 & 1.98 & 2.63 & $71.2-79.3$ \\
$\gamma$ & 4.93 & 0.362 & 7.34 & $4.18-5.67$ \\
\hline
\end{tabular}

microsomes. Glucuronides of Indole 15 were not observed. A variety of phase I and phase II metabolites of Indole 15 were detected in the biological excreta. These metabolites indicate that Indole 15 is susceptible to two major metabolism pathways: oxidation and sulfate conjugation. Future compounds incorporating a variety of functional groups (e.g., electron donating and withdrawing groups) in the indole ring and/or the benzyl ring, may represent a reasonable approach to reduce oxidative metabolism and maintain anticancer activity, providing more metabolically stable compounds.

In order to address the mechanistic features of pharmacological systems for anticancer agents, an approach involving time-dependent transduction processes $(6,11,12)$ is often applied to describe tumor growth kinetics. The threshold concentration for tumor eradication $\left(\mathrm{C}_{\mathrm{T}}\right)$ was described as the drug concentration required to fully inhibit tumor growth (4). When first-order tumor growth is assumed, $\mathrm{C}_{\mathrm{T}}$ is then defined as the ratio of two rate constants, the growth rate over the killing rate $(\mathrm{kg} / \mathrm{k})$. The sulforhodamine B (SRB) assay was used to assay cell growth inhibition by Indole 15 in vitro using PC-3 cells. The concentration-cell growth profile was well described by a sigmoid model using nonlinear least squares method with the procedure PROC NLIN (SAS version 9.1, ASA Institute, Cary, NC, USA). About $10 \%$ of PC-3 cells remained alive at very high concentrations 
of Indole 15 (Fig. 8), although the $\mathrm{IC}_{50}$ was relatively small $(75.3 \mathrm{nM})$ (Table V). Based on the parameters estimated in the transduction model, $\mathrm{C}_{\mathrm{T}}$ (the ratio of $\mathrm{kg} / \mathrm{k}$ ) is calculated as $0.94 \mu \mathrm{g} / \mathrm{ml}$ (equivalent to $2.8 \mu \mathrm{M}$ ), a value consistent with the in vitro cell cytotoxicity study.

Simulations based on the developed pharmacokinetic and pharmacodynamic model were further conducted to predict PC-3 tumor growth under different dosing regimens, i.e., BID vs. QD at different doses. The results suggest that the percentage of tumor reduction is only dependent on Indole 15 dose level, regardless of the dosing regimens (data not shown). Therefore, our late studies with Indole 15 focused on strategies to identify the lowest effective dose, improve oral bioavailability and reduce toxicity. Overall, these studies provided a mechanistic understanding of the factors governing the in vivo anticancer activity of Indole 15 and served as the foundation for subsequent studies showing that Indole 15 is a member of a new class of orally bioavailable tubulin antagonists.

\section{Acknowledgements}

This study was supported by a grant from NIH R01 DK 065227-01. Sunjoo Ahn, Duane D. Miller and James T. Dalton are employees of GTx Inc. and hold stock options in the company.

\section{References}

1. Ahn S, Hwang DJ, Barrett CM, et al: A novel bis-indole destabilizes microtubules and displays potent in vitro and in vivo antitumor activity in prostate cancer. Cancer Chemother Pharmacol 67: 293-304, 2011.
2. Lu Y, Li CM, Wang Z, et al: Design, synthesis, and SAR studies of 4-substituted methoxylbenzoyl-aryl-thiazoles analogues as potent and orally bioavailable anticancer agents. J Med Chem 54: 4678-4693, 2011.

3. Dethlefsen LA, Prewitt JM and Mendelsohn ML: Analysis of tumor growth curves. J Natl Cancer Inst 40: 389-405, 1968.

4. Simeoni M, Magni P, Cammia C, et al: Predictive pharmacokinetic-pharmacodynamic modeling of tumor growth kinetics in xenograft models after administration of anticancer agents. Cancer Res 64: 1094-1101, 2004.

5. Magni P, Simeoni M, Poggesi I, et al: A mathematical model to study the effects of drugs administration on tumor growth dynamics. Math Biosci 200: 127-151, 2006.

6. Yang J, Mager DE and Straubinger RM: Comparison of two pharmacodynamic transduction models for the analysis of tumor therapeutic responses in model systems. AAPS J 12: $1-10,2010$.

7. D'Argenio DZ and Schumitzky A: ADAPT II User's Guide: Pharmacokinetic/Pharmacodynamic Systems Analysis Software: Biomedical Simulations Resource, Los Angeles, CA, 1997.

8. Davies B and Morris T: Physiological parameters in laboratory animals and humans. Pharm Res 10: 1093-1095, 1993.

9. Abbara C, Rouchon C, Hosten B, et al: Enhanced oral bioavailability of paclitaxel by recombinant interleukin-2 in mice with murine Lewis lung carcinoma. Drug Metabol Drug Interact 20: 219-231, 2004

10. Eiseman JL, Eddington ND, Leslie J, et al: Plasma pharmacokinetics and tissue distribution of paclitaxel in CD2F1 mice. Cancer Chemother Pharmacol 34: 465-471, 1994.

11. Mager DE and Jusko WJ: Pharmacodynamic modeling of time-dependent transduction systems. Clin Pharmacol Ther 70: 210-216, 2001.

12. Sun YN and Jusko WJ: Transit compartments versus gamma distribution function to model signal transduction processes in pharmacodynamics. J Pharm Sci 87: 732-737, 1998. 